\title{
Improvements in Serial Crystallography Capabilities at GM/CA
}

\author{
${ }^{1}$ Kissick, D. J., ${ }^{2}$ Martin-Garcia, J.M., ${ }^{2} \mathrm{Hu}, \mathrm{H} .,{ }^{1}$ Venugopalan, N., ${ }^{1} \mathrm{Xu}, \mathrm{S} .,{ }^{1}$ Corcoran, S., \\ ${ }^{1}$ Ferguson, D., ${ }^{1}$ Hilgart, M. C., ${ }^{1}$ Makarov, O., ${ }^{1}$ Xu, Q., ${ }^{1}$ Ogata, C., ${ }^{1}$ Stepanov, S., ${ }^{2}$ Thifault, D., \\ ${ }^{2}$ Marlowe, T., ${ }^{2}$ Alvarado, C., ${ }^{2}$ Zacks, M., ${ }^{2}$ Cance, W., ${ }^{2}$ Fromme, P., ${ }^{1}$ Fischetti, R. F. \\ ${ }^{1}$ Advanced Photon Source, Argonne National Laboratory, Lemont, IL 60439 (dkissick@anl.gov) \\ ${ }^{2}$ Biodesign Institute, Arizona State University, Tempe, AZ 85287
}

Successful proof-of-concept experiments have demonstrated the feasibility of synchrotron serial crystallography at GM/CA ${ }^{1}$. Recent hardware and software upgrades will allow routine user operation of serial data collection. Beam shape and intensity have been improved by the addition of compound refraction lenses (CRL). The CRLs provide nearly 10 times higher photon flux than the proof-ofconcept measurements, allowing data collection at $100 \mathrm{~Hz}$. All the components for high-viscosity injector-based sample delivery are installed in the ID-D endstation and these components can be setup during the normal user startup time ( $1 \mathrm{hr})$. During injector-based experiments, longer beam collimators and a tapered beam stop can be used to decrease background noise by a factor of $\sim 10$ by reducing air scatter before and after the sample. Fixed samples can generate serial datasets as well using a modified raster scan that allows sample rotation. The software suites Cheetah and CrystFEL as well as in house software allow real-time monitoring, data reduction, and processing. Early April 2019 marked the first injector-based serial crystallography experiments at GM/CA where the goal was to determine a novel protein structure. Serial crystallography data were collected for various samples including lysozyme, proteinase K, and focal adhesion kinase 919 (FAT-919). We have determined the structure of lysozyme to $1.55 \AA$ resolution and the structure of FAT-919 to $2.5 \AA$ resolution.

[1] Martin-Garcia, J. M., et al. (2017). IUCrJ 4, 439-454. https://doi.org/10.1107/S205225251700570X 